\title{
Detecting and Preventing "Multiple-Account" Cheating in Massive Open Online Courses
}

\author{
Curtis G. Northcutt \\ Massachusetts Institute of Technology, Cambridge, MA 02139, USA \\ cgn@mit.edu \\ Andrew D. Ho \\ Harvard Graduate School of Education, Cambridge, MA 02138, USA \\ andrew_ho@gse.harvard.edu \\ Isaac L. Chuang \\ Massachusetts Institute of Technology, Cambridge, MA 02139, USA \\ ichuang@mit.edu
}

August, 2015

Computers \& Education

Please direct correspondence to Curtis G. Northcutt at cgn@mit.edu. This material is based upon work supported by the National Science Foundation Graduate Research Fellowship under Grant No. (\#1122374). The work presented is the sole work of the authors. The authors assume full responsibility for the contents of this manuscript. 


\title{
Detecting and Preventing "Multiple-Account" Cheating in Massive Open Online Courses
}

\begin{abstract}
:
We describeacheating strategy enabled by the features of massive open online courses (MOOCs) and detectable by virtue of the sophisticated data systems that MOOCs provide.The strategy, Copying Answers using Multiple Existences Online ${ }^{1}$ (CAMEO), involves a user who gatherssolutions to assessment questionsusing a "harvester" account and then submits correct answers using a separate "master" account.We use a small-scale experiment to verify CAMEO and estimate a "lower bound" for its prevalence among1.9 million course participantsin 115MOOCs from two universities.Using conservative thresholds, we estimate CAMEO prevalence at 1,237 certificates, accounting for $1.3 \%$ of the certificates in the 69 MOOCs with CAMEO users. Among earners of 20 or more certificates, $25 \%$ have used the CAMEO strategy. CAMEO users are more likely to be young, male, and international than other MOOC certificate earners. We identify preventive strategies that can decrease CAMEO rates and show evidence of their effectiveness in science courses.
\end{abstract}

\section{KEYWORDS:}

Massive Open Online Courses (MOOCs); Cheating Detection; Educational Certification; Educational Data Mining (EDM); Security

\section{C\&E KEYWORDS:}

architecture for educational technology system; learning communities; lifelong learning; pedagogical issues; teaching/learning strategies

\footnotetext{
${ }^{1}$ CAMEO is an abbreviation for Copying Answers using Multiple Existences Online
} 


\section{Introduction and Motivation}

Massive Open Online Courses (MOOCs) began receiving significant media coverage in 2012 (Pappano, 2012; McNutt, 2013), coincident with the widespread commitment byestablished universities to providing free courses online (Ho et al., 2014; Christensen, Steinmetz, Alcorn, Bennett, Woods, \& Emanuel, 2013; Stanford Online, 2013). These MOOCs distinguished themselves from predecessors like MIT's Open Courseware (d'Oliveira, Carson, James, \& Lazarus, 2010; Smith, 2009) by providing not only free content but a course-like structure, including enrollment, synchronous participation, periodic graded assessments, online discussion forums, interactive simulations, and of greatest relevance for our purposes, certification of successful completion (DeBoer, Ho, Stump, \&Breslow, 2013; Linn, Gerard, Ryoo, McElhanney, Liu, \&Rafferty; 2014).One theory of MOOC proliferation holds that free certification of proficiency in college courses can reduce inefficiencies in higher education by replacing highcost residential courses with low-cost online certification (Hoxby, 2014).

In this paper, we reveala particular cheating strategy that is detectableacross the $115 \mathrm{MOOCs}$ in our sample and currently presents a serious threat to the trustworthiness of theircertifications.We call the strategy, Copying Answers using Multiple Existences Online (CAMEO). A user employing this strategy, whom we refer to as a CAMEO user, earns a certificate by creating at least two MOOC accounts: (1)one or more "harvester" accountsused to acquirecorrect answers by guessing at test answers and then accessing instructor-provided solutions via a "Show Answer" button, and (2)one or more"master" accountsused to submitthese solutions as correct test answers.

The CAMEO strategy lies at the intersection of a number of other copying techniques and contexts. We distinguish between 1) what is copied, 2) why it is copied, 3) how it is copied, and 4) how copying is detected.The CAMEO strategy occurs in similar contexts as community collaboration in online courses (Yang, Wen, Kumar, Xing, \& Rose, 2014), and detection of both involves analyzing the interactions of multiple accounts. However, prior efforts have focused on how communities of different users affect learning outcomes (Kumar, Rose, Wang, Joshi, \& Robinson, 2007), in contrast with CAMEO behavior, where a single user exploits multiple accounts, potentially circumventing the learning process entirely. CAMEOis most similar to 
"multiple account" sharing strategies in online games (e.g., Kafai \& Fields, 2009), where a single user can increase scores or other in-game outcomes by creating multiple accounts and interacting them strategically. However, CAMEO behavior distinguishes itself from online game strategies due to what is copied (correct answers to tests) and why it is copied (to fake or expedite certification of proficiency). As we show, the specificity of these differences enables targeted detection, quantification, and prevention of CAMEOuse in these MOOCs.

Cheating by CAMEO shares similarity in purpose withcopying in online and conventional courses(McCabe, Butterfield, \& Treviño, 2012; Palazzo, Lee, Warnakulasooriya, \& Pritchard, 2010; Baker, Corbett, \& Koedinger, 2004; Kauffman \& Young, 2015). However, three features of CAMEO make it a unique threat as a cheating strategy in online education. First, it is internally sufficient. Whereas most users copy from other students or external resources, CAMEO users employ multiple accounts to copy from themselves, makingthe cheating strategy highly accessible by removing dependence on outside resources. As a result, the strategy is extremely effective.Second, in asynchronous MOOCs, where students can access course materials and assessments at their own pace, a CAMEO user can employ the CAMEO strategy for every question they attempt, allowing certification for full course completion in a single sitting. Third, it is unrestricted, employable in a nonselective, open admission setting. Degrees from selective institutions assert, at the very least, that users have been pre-screened, but MOOC certificates do not. Because MOOC users, unlike most postsecondary students, are not selected by any merit-based process or criteria, the considerable accessibility of CAMEO in these MOOCs holds the potential to render their certificates valueless as an academic credential.

Thekey contributions of this paper are a detection algorithm for the CAMEObasedcheating that allows for a lower bound estimate of prevalence and a small-scale experiment confirming CAMEO behavior. This latter experiment is an extension of "honey pot" cheating detection (Corrigan-Gibbs, Gupta, Northcutt, Cutrell, \& Thies, 2015), where copied answers can be confirmed directly. These contributions complement the considerable literature that estimates cheating prevalence through surveys, where survey responses may be influenced by social desirability, interpretation of item prompts, concerns about anonymity, and inflation in selfreported performance (Mastin, Peszka, \& Lilly, 2009). This paper investigates a specific cheating strategy using analgorithm customized to big datasets that containdetailed user interactions with online course content, including activity timestamps. With 115 courses, this is also the largest 
analysis of cheating in online courses of which we are aware.

CAMEO also represents an example of a more general tendency for open online learning systems to enable both new strategies for cheating and new strategies for detection (Horton,

Rand, \& Zeckhauser, 2011; Li, Chang, Yuan, \& Hauptmann, 2015; Raines et al., 2011).Although CAMEO is technically a copying strategy, we argue that its use in MOOCs constitutes “cheating."At a minimum, employing CAMEO is a violation of policy, because MOOC honor codes forbid the creation of multiple accounts (Coursera, 2012; edX, 2014; Udacity, 2014). The CAMEO strategy also threatens perceptions of the value of MOOC certification. Any reasonable interpretation of standard MOOC certificates, whichrefer to "successful completion" (edX, 2015),includes proven student proficiency with course content. Yet, the prevalence ofthe CAMEO strategyjustifies a starkly contrasting interpretation of MOOC certification - that a user merely copied answers from a“dummy"harvester account. Combined with growing evidence that the reputation and usefulness of MOOC certification are predictors of MOOC persistence (e.g., Alraimi, Zo, \& Ciganek, 2015), we anticipate that widespread awareness of MOOC susceptibility to the CAMEO strategy could depress MOOC popularity and persistence among general users.

\section{Methodology}

We begin by describing a CAMEO detection algorithm that relies on the distribution of differences in time between particular user actions across particular user pairs. The CAMEO detection algorithm is comprised of five filters with highly conservative cutoffs intended to reduce false positives, including a Bayesian criterion for the timestamp difference distributions. After we present these filters, we describe a small-scale experiment that confirms CAMEO cases, and we show that the CAMEO algorithm detects these cases as expected.

\subsection{Indicators of "Copying Answers using Multiple Existences Online” (CAMEO)}

Fig.1 illustrates two prototypical CAMEO users, each with two accounts, and their timeline of interactions with online assessments. For both CAMEO usersin Fig.1, we also illustrate the variable: 


$$
\Delta t_{m, h, c, i}=t_{m, c, i}-t_{h, c, i}
$$

This is the difference between the time that a master account, $m$, submits a correct answer and the time that a harvester account, $h$, acquires the correct solution, for a problem (item) in common, $i$, in a given MOOC course, $c$. It is possible for a single master to have multiple harvesters and a single harvester to have multiple masters. The subscript, $c$, recognizes that the same masterharvester pair may be employing CAMEO across multiple courses.

Logically, for CAMEO users, these $\Delta t$ are predominantly or entirely positive in sign. The former time, $t_{m, c, i}$, is recorded in server log files. For the latter time, $t_{h, c, i}$, we take advantage of the fact that instructors of the MOOCs in our sample generally allow users to click a "show answer" option after submitting answers, to display a staff-prepared answer and/or an explanation of the solution, in order for users to obtain rapid feedback. The timestamp produced by a "show answer" clickdefines- $t_{h, c, i}$. We introduce a method for probabilistic detection of CAMEO users based on observed distributions of $\Delta t_{m, h, c}$ over itemsi.

\subsection{Detection of "Copying Answers using Multiple Existences Online" (CAMEO)}

The detection strategy begins by considering all possible ordered pairs of accounts, within each course, as candidate CAMEO users. It asks whether the pattern of "show answers" from one, the "candidate harvester" $(\mathrm{CH})$, and "correct answers" from the other, the "candidate master" (CM), is ordered and coincident enough to declare the CH-CM pair a CAMEO user. In total, we employ five filters to identify CAMEO users (Table 1). These five filters are conjunctive and thus order-independent; we group them conceptually and order them narratively.

The first two filters reflect the logic that a CAMEO user's $\mathrm{CH}$ often provides correct answers totheCM fairly quickly; thus, the distribution of $\Delta t_{m, h, c}$ over items $i$ should be positive with small magnitudes.Fig. 2 shows four contrasting distributions of $\Delta t_{m, h, c}$ for four different $\mathrm{CH}$ CM pairs. Distribution A illustrates two unrelated and asynchronous accounts, where one user's "show answer" event is sometimes before and sometimes after another user's correct answer submissions by times that vary widely in magnitude; distributions like this should be common. Distribution B illustrates two users (e.g. siblings, roommates, or students taking the assessment side-by-side) working in close synchronicity. Due to chance and differences in pacing, one user's 
"show answers" will sometimes precede but sometimes follow the other's "correct answers," but times will be in close proximity.

Distribution C reflects prototypical CAMEO behavior, corresponding to Fig. 1. All $\Delta t_{m, h, c}$ are positive, and their magnitudes are extremely small, centered in this illustration at around 10 seconds. These small $\Delta t_{m, h, c}$ magnitudes are typically possible when the CAMEO user is logged in simultaneously to both $\mathrm{CH}$ and $\mathrm{CM}$ accounts on different internet browsers or computers. Finally, Distribution $\mathrm{D}$ is also positive but with $\Delta t_{m, h, c}$ magnitudes that are larger and more variable. This is consistent with ordered coincidence, where unrelated pairs of users will be offset from each other due to different enrollment dates or time-of-day preferences.

To identify CAMEO users by distributions of $\Delta t_{m, h, c}$, we consideredconstraining the population distribution of $\Delta t_{m, h, c}$ or $\left|\Delta t_{m, h, c}\right|$ by strong parametric assumptions (e.g., log-normal, exponential),but many observed distributions had extreme skew due to outlying $\Delta t_{m, h, c, i}$ values. We therefore opt for a less parametric approach that targets the percentage of positive observations (Filter 1) and the magnitude of the $90^{\text {th }}$ percentile (Filter 2).

\subsubsection{Filter 1 and the Bayesian criterion}

For Filter 1, given variation in the quantity of data shared between any $\mathrm{CH}$ and $\mathrm{CM}$, we use a Bayesian criterion that is more stringent when data are limited (Lehmann \& Casella, 1998).We estimate the parameters of the posterior distribution of a proportion $\pi$, our parameter of interest indicating the proportion of positive $\Delta t_{m, h, c, i}$ values, given $n$, as the number of in-common items for which a $\mathrm{CH}$ has a "show answer" and a CM has a correct answer, and $x$, as the number of times that the $\mathrm{CH}$ time precedes the $\mathrm{CM}$ time:

$$
x_{m, h, c}=\Sigma_{\mathrm{i}=1: \mathrm{n}} I\left(\Delta t_{m, h, c, i}>0\right)
$$

Here, Iis the indicator function, which is 1 when the argument is true and 0 otherwise. The maximum $n$ for any CH-CM pair is the number of items. The average number of graded items is 141 , across courses, allowing considerable data for inference. We assume that xis binomially distributed and that $\pi$ has a Beta distribution. Following standard rules of conjugacy:

$$
\begin{aligned}
& x \mid n, \pi \sim \operatorname{Binomial}(\pi, n) \\
& \pi \mid \alpha, \beta \sim \operatorname{Binomial}(\pi, n) \\
& \pi \mid x, n, \alpha, \beta \sim \operatorname{Beta}(\alpha+x, \beta+n-x)
\end{aligned}
$$


We observe $x$ and $n$ in the data. For the prior distribution, we $\operatorname{set} \alpha=\beta=0.5$, empirically and judgmentally, using full distributions of observed $p=x / n$ when $n$ is large in our data. This is a gentle U-shape, consistent with the fact that many distributions of $t_{m, c}$ are stochastically or entirely offset from other distributions of $t_{h, c}$ in one direction or other, due to the asynchronous nature of MOOCs.

We operationalize Filter 1 in terms of confidence that $\pi$ is close to 1 , that is, that $\mathrm{CH}$ interaction with an item almost always precedes CM interaction. Specifically, Filter 1 selects $\mathrm{CH}-\mathrm{CM}$ pairs with a $90 \%$ probability of $\pi_{m, h, c}>0.9$. This is a conservative, stringent criterion that requires considerable data before concluding that a distribution is predominantly positive. Even a $\mathrm{CH}-\mathrm{CM}$ pair with $x=12$ out of $n=12(p=100 \%)$ positive values is insufficient to meet this criterion.

\subsubsection{Filter 2 and setting the cutoff threshold}

Filter 2 addresses the fact that Filter 1 excludes Distributions A and B from CAMEO consideration, but it cannot distinguish between Distributions C and D (Fig. 2). To exclude ordered accounts that happen to be offset in time in the positive direction, Filter 2 uses the $90^{\text {th }}$ percentile of the $\Delta t_{m, h, c}$ distribution as a criterion, setting a conservative cutoff at 5 minutes. In other words, $90 \%$ of the $\Delta t_{m, h, c}$ values must be less than 5 minutes. This cutoff occurs at an “elbow"as shown in Fig. 3,where shifting the cutoff between 0 and 5 minutes changes the number of estimated CAMEO users dramatically, and subsequent shifts past 5 minutesdo not.

\subsubsection{Filter 3: certified $C M-$ uncertified $C H$ pairs.}

The first two filters provide considerable evidence that, for CAMEO users, the distribution of $\Delta t_{m, h, c}$ is disproportionately positive and centered at less than 5 minutes in time. Filters 3 through 5 provide convergent criteria to further minimize the probability of false identification.

Filter 3 considers only $\mathrm{CH}-\mathrm{CM}$ pairs for which the $\mathrm{CH}$ is uncertified and the $\mathrm{CM}$ is certified. Although this may discard CAMEO users who do not ultimately earn certification, our intention is to address possible threats to MOOC certificate validity as directly as possible, so we include only certified CMs. In addition, a $\mathrm{CH}$ that earns a certificate is inconsistent with the interpretation of CAMEO users as a cheating strategy, since it leaves open the possibility that the 
$\mathrm{CH}$ is actually proficient in the course.

\subsubsection{Filter 4 and Detecting Shared IP address}

Filter 4 further reduces the candidate pool to those $\mathrm{CH}-\mathrm{CM}$ pairs who share an IP address, defined for each account as the modal (most commonly used) IP address across all logged interactions in a given course $c$. However, considering only users with the same IP address fails to detect users who employ the CAMEO strategy using accounts assigned different modal IP addresses in a given course, either by coincidence or intentional misdirection. To improve detection of these users, we broaden the definition of "sharing an IP address" to CH-CM pairs who have ever shared an IP address in their course-taking history.

To detect CAMEO userswithaccounts having different modal IP addresses in a given course, we consider every unique (name, IP) tupleacross all accounts participating in any of the 115 courses analyzed. We assign each (name, IP) an "IP group", initially as a unique integer for each pair. Next, we group by modal IP address such that all (name, IP) tuplessharing the same modal IP address are assigned (merged into) the same IP group. Then, we group by username such that all (name, IP) tuplessharing the same username are merged into the same IP group. We repeat both the "merge by IP" and "merge by username" steps until the IP group no longer changes. This can be described as a "transitive closure" of modal IP address and account namesfor all accounts across courses. It allows us to consider $\mathrm{CM}-\mathrm{CH}$ pairs whenever the two accounts have shared a common modal IP address within a course, across courses, or across other accounts that have shared the same modal IP address within and across courses.

\subsubsection{Filter 5: excluding shared routers}

Filter 5 excludes all $\mathrm{CH}-\mathrm{CM}$ pairs who are part of a group that has 10 accounts or more that share a modal IP address. We intend this to exclude shared routers among classrooms or cafes that might increase the likelihood of false positives.

\subsection{Verification of "Copying Answers using Multiple Existences Online" (CAMEO)}

We conducted a small-scale, targeted investigation of registrants in a single, small course to confirm existence of the CAMEO strategy. Through descriptive analyses of usage patterns over time, the instructor identified 3 pairs of users, consisting of 3 candidate master accounts and 
3 candidate harvester accounts, whose assessment submissions seemed unusually synchronous.For these three user pairs, we adapted answers to 7 test questions to append a unique random string to the answer displayed to each user.This string took the form of a superfluous symbol (e.g. parentheses), negligible decimal points at the end of a correct answer, or an expression that evaluates to 1. For example, an answer to the question "what is the final momentum of the particle?"could be 3.13, butthe answer was displayed as "3.13556" to one user, and 3.13417" to another.For logistical and pedagogical reasons, this targeting was restricted to these three user pairs.

One of these three pairs never viewed these items. For both of the remainingcandidate master accounts, we detect direct copying of at least one unique answer from the harvester accounts. This confirms CAMEO behavior, given that the unique combinations of extra digits and symbols had no reason to be submitted and could not have happened by chance. For small-scale validation, among the 3 pairs of users, the CAMEO detection algorithm identified only and exactly the same two master accounts as CAMEO users. The next section builds from this existence proof to estimate the lower bound prevalence for CAMEO behavior in these MOOCs.

\section{Results}

We investigate the prevalence of CAMEO users in 115 online courses from two institutions, Harvard University and MIT, offered on the MOOC platform, edX. ${ }^{2}$ We use data from courses from the fall of 2012 through the spring of 2015, up to an analytic cutoff date of June 2, 2015. About half of these MOOCs are described in detail in other reports (McNutt, 2013; Ho et al., 2015) that emphasize their range of curricular foci and their heterogeneous participant demographics. Our sample consists of 1,893,092 enrollments (1,067,570 from unique accounts) whose users clicked into the course content at least once. A total of 155,301 certificates were ultimately earned from 103,370 unique accounts.

\subsection{Prevalence of CAMEO}

\footnotetext{
${ }^{2} \mathrm{~A}$ list of the 115 courses studied, with their classifications into topic areas, and $\Delta t_{m, h, c}$ distribution data for CM-CH pairs, are archived in the Harvard Dataverse Network, at http://dx.doi.org/10.7910/DVN/3UKVOR.
} 
Across these courses, we estimate that a total of 1,237 certificates were earned using the CAMEO strategy, 1\% across all 115 courses, by 657 unique users employing 674 harvester accounts. In some courses, CAMEO users account for as many as $5 \%$ of the certificates earned. Across the 69 courses in which we identified CAMEO users, they account for $1.3 \%$ of certificates. Table 2A shows that CAMEO users are more likely to be young, male, less educated, and international than their certified counterparts in the same courses(Ho et al., 2015).Among countries with at least 20 CAMEO users, countries with the highest CAMEO counts per certificates were Albania (12\%), Indonesia (4\%), Serbia (3\%), Colombia (2\%), and China (2\%). The CAMEO rate in the USA is particularly low, at $0.4 \%$ of certificates earned.Table 2B shows CAMEO prevalence by broad curricular area. Prevalence of CAMEO users is greatest in the Government, Health, and Social Science category $(1.3 \%)$ and lowest in the Computer Science category $(0.1 \%)$.

\subsection{Prevention of CAMEO}

Mechanisms which logically prevent CAMEO use include restricting the "show answer" option until after assignments are due, and using algorithmic generation of assessment items so that participants receive randomly varying items, each with different solutions. Across the 37 Science, Technology, Engineering, and Mathematics (STEM) courses in this sample, 18 employed such prevention mechanisms. Table $2 \mathrm{C}$ shows that the CAMEO rate in courses that employed these preventive strategies in half or more of the assessment items was substantially lower $(0.1 \%)$ than the rate in courses that did not employ preventive strategies $(1.2 \%)$.

\section{Discussion}

As open online courses proliferate, we identify CAMEO as a significant threat to the validity of large-scale certification. Our primary goals are to demonstrate that CAMEO exists and to bound its prevalence in the population. We believe that our method accomplishes this and does so conservatively. Nonetheless, we raise here a central shortcoming of this work and address it briefly while encouraging subsequent research. Like many cheating analyses in real contexts, we have no "true" knowledge of cheating to evaluate whether our detection method is 
accurate at the individual level. Perhaps a child is guessing haphazardly and clicking "show answer," while working with a parent who separately submits answers correctly, always a few minutes after the child. This is unlikely but not impossible. However, our aim is not to identify individuals but estimate aggregate prevalence. We believe our filters, combined with the smallscale experiment that provides an existence proof, accomplish this.

We also raise three convergent sources of evidence. First, text-matching of usernames reveals considerable overlap in candidate pairs;many CAMEO users have usernames consistent with the Master-Harvester hypothesis, like "Curtis1" and "Curtis2." Second, although our CAMEO detection algorithm treats every $\mathrm{CM}-\mathrm{CH}$ pair independently, we find CAMEO behavior is clustered within users. A total of 43 separate accounts have earned 5 or more certificates by CAMEO. Third, we conducted a limited analysis, in one course, of plagiarism by copying openresponse text across users, and we find that these accounts are also identified as CAMEO users. Although we believe our algorithm alone is sufficient to demonstrate the existence and bound the prevalence of CAMEO, we encourage further research to support validation of the detection algorithm.

Another concern is the possibility that some users could be using CAMEO to increase their exposure to assessment items and thereby increase their learning. We argue that this is unlikely given how we operationalize our definition. CAMEO users require nearly all of $\mathrm{CH}$ "show answer" clicks to occur "shortly" before CM correct answer submissions. In fact, we found that often the actual time difference was only a few seconds. The extent and timing of this systematic behavior is most consistent with a cynical and blatant attempt to harvest correct answers to rapidly acquire certification, not with a learning strategy.

Finally, although this CAMEO algorithm takes advantage of assessment features in these particular courses on this particular MOOC platform, CAMEO, as a general multiple-accountcopying strategy, is possible in any MOOC with open signup policies. Generalization of the approach and its conclusions is certainly possible though arguably less scalable. Many of the courses we analyze use assessment approaches that do not involve or circumvent the "show answer" flag. From this perspective, CAMEO rates in these courses are underestimates of true CAMEO rates, and our algorithm would have to be tuned to the particular environments of these courses. For example, in an independent study tailored to a single course(Alexandron, Ruipérez- 
Valiente, \& Pritchard, 2015), 9.8\% of certificate earners were identified as harvesting at least one answer.

Our estimates of cheating prevalence are arguably consistent with higher estimates from surveys. Such surveys typically ask a variant of thequestion "Have you cheated?" with allowance forrecency and magnitude (McCabe, Butterfield, \& Treviño, 2012). In contrast, CAMEO is complete in its scope and course-specific, as the introduction notes. The analogous question we address is, "Did you cheat your way through this entire course?"We can establish a basis for comparison through the observation from our data, that those who certify in multiple courses are much more likely to have used the CAMEO strategy at least once, including $25 \%$ of those who have earned at least 20 certificates, as depicted in Table 3 . We consider this commensurate in severity to the reports that two-thirds of college students have engaged in some form of academic dishonesty in the previous year (McCabe, Butterfield, \& Treviño, 2012), especially considering that the minimum threshold in our analysis is sufficient cheating to earn certification, versus being dishonest in just one or a few problems.

Our findings are consistent with other observations that MOOC assessment infrastructures rarely support robust inferences about learning (Reich, 2015). All feasible mechanisms that prevent the CAMEO strategyhave a downside. If instructors withhold the "show answer" option until after the problems are graded, this would constrain generally desirable asynchronous MOOC usage, and students will not have the rapid feedback touted as a pedagogical benefit of online learning environments. Algorithmic generation of assessment items and correct answers is challenging and only suitable for some subjects and assessment tasks.

Beyond honor codes (LoSchiavo \& Shatz, 2011; Corrigan-Gibbs, Gupta, Northcutt, Cutrell, \& Thies, 2015), a solutionembraced by many MOOC purveyors (Kolowich, 2013; Straumsheim, 2015; Eisenberg, 2013) is to offer certificates earned under controlled assessment conditions, such as in-person assessments taken at secure testing centers for a fee. We observe that the cost and constraints associated with fee-based, in-person testing centers are antithetical to the open, online principles that define MOOCs, as well as their mission of improving worldwide access to not just learning but certification opportunities. Further research on cheating detection and prevention, including experiments that can isolate factors that cause and discourage cheating, is necessary to design spaces and structures that can support openand trustworthy certification at scale. 


\section{Conclusion}

The CAMEO detection algorithm uses three strategies that hold general promise for the analysis of clickstream data. First, time difference analysis is a tool to infer relationships among students. Second, Bayesian criteria allow appropriately conservative classification when data are limited. Third, transitive closure is a technique for robust consideration of possible CAMEO users. Beyond cheating detection in MOOCs, these tools may aid more generally in identification of collaboration and interaction among online users.

Thereis continued interest in the potentialfor MOOCs to increase efficiency and spur innovation in higher education. Four features of CAMEO severely undermine this potential. First, unless prevented, this cheating strategy allows students to earn certificates in open online courses without any understanding of the domain material. Second, the strategy is highly convenient, requiring no interactions with external resources, either animate or inanimate. Third, it is unrestricted, employable in a nonselective, open admission setting. Fourth, whereas cheating is traditionally considered with respect to individual assessments or portions thereof, CAMEO is a course-level strategy. It is less cheating than the wholesale falsification of a certificate.

In this paper, we have demonstrated the prevalence of the CAMEO cheating strategy in a large sample of MOOCs, and we have argued that it poses a serious threat to interpretations of theircertifications. Protecting certification requires CAMEO prevention, and we have shown that preventive strategies hold promise. Yet, CAMEO is only one of many possible cheating strategies. Sophisticated detection algorithms should be a part of a general approach to protect the validity of online course certification. We recommend and look forward to future interventions that increase and encourage honest behavior in online learning environments while disallowing and discouraging cheating in all its forms.

\section{Acknowledgements}

Omitted to ensure anonymity for reviewers. 


\section{References}

Alexandron, G., Ruipérez-Valiente, J. A., \& Pritchard, D. E. (2015). Evidence of MOOC Students Using Multiple Accounts to Harvest Correct Answers. Learning with MOOCs II.

Alraimi, K. M., Zo, H., \& Ciganek, A. P. (2015). Understanding the MOOCs continuance: The role of openness and reputation. Computers \& Education, 80, 28-38.

Baker, R. S., Corbett, A. T., \& Koedinger, K. R. (2004, January). Detecting student misuse of intelligent tutoring systems. In Intelligent tutoring systems(pp. 531-540). Springer Berlin Heidelberg.

Christensen, G., Steinmetz, A., Alcorn, B., Bennett, A., Woods, D., \& Emanuel, E. J. (2013). The MOOC phenomenon: who takes massive open online courses and why? http://dx.doi.org/10.2139/ssrn.2350964

Corrigan-Gibbs, H., Gupta, N., Northcutt, C., Cutrell, E., \& Thies, W. (2015, March). Measuring and Maximizing the Effectiveness of Honor Codes in Online Courses. Proceedings of the Second (2015) ACM Conference on Learning@Scale (pp. 223-228). ACM. http://dx.doi.org/10.1145/2724660.2728663

Corrigan-Gibbs, H., Gupta, N., Northcutt, C., Cutrell, E., \& Thies, W. (2015, December). Deterring Cheating in Online Environments. ACM Transactions on Computer-Human Interaction (TOCHI).

Coursera. (2012, April). Terms of Use, Privacy Policy and Honor Code. Retrieved from https://authentication.coursera.org/auth/auth/normal/tos.php

DeBoer, J., Ho, A. D., Stump, G. S., \& Breslow, L. (2014). Changing “Course” Reconceptualizing Educational Variables for Massive Open Online Courses. Educational Researcher. http://dx.doi.org/10.3102/0013189X14523038

d'Oliveira, C., Carson, S., James, K., \& Lazarus, J. (2010). MIT OpenCourseWare: Unlocking knowledge, empowering minds. Science, 329(5991), 525-526.

edX. (2014, October 22). edX Terms of Service. Retrieved from https://www.edx.org/edx-terms-service

edX. (2015). Student FAQ. Retrieved from https://www.edx.org/about/student-faq

Eisenberg, A. (2013, March 2). Keeping an eye on online test-takers. The New York Times. Retrieved from http://www.nytimes.com/2013/03/03/technology/new-technologies-aim-to-foil-online-course-cheating.html

Ho, A. D., Reich, J., Nesterko, S. O., Seaton, D. T., Mullaney, T., Waldo, J., \& Chuang, I. (2014). HarvardX and MITx: The first year of open online courses, Fall 2012-Summer 2013. http://dx.doi.org/10.2139/ssrn.2381263

Ho, A. D., Chuang, I., Reich, J., Coleman, C. A., Whitehill, J., Northcutt, C. G., ... \& Petersen, R. (2015). HarvardX and MITx: Two Years of Open Online Courses Fall 2012-Summer 2014. http://dx.doi.org/10.2139/ssrn.2586847

Horton, J. J., Rand, D. G., \& Zeckhauser, R. J. (2011). The online laboratory: Conducting experiments in a real labor market. Experimental Economics, 14(3), 399-425.

Hoxby, C. M. (2014). The economics of online postsecondary education: MOOCs, nonselective education, and highly selective education (No. w19816). National Bureau of Economic Research.

Kafai, Y. B., \& Fields, D. A. (2009). Cheating in virtual worlds: Transgressive designs for learning. On the horizon, 17(1), 12-20. http://dx.doi.org/10.1108/10748120910936117

Kauffman, Y., \& Young, M. F. (2015). Digital plagiarism: An experimental study of the effect of instructional goals and copy-and-paste affordance. Computers \& Education, 83, 44-56.

Kolowich, S. (2013, April 15). Behind the Webcam's Watchful Eye, Online Proctoring Takes Hold. The Chronicle of Higher Education. Retrieved from http://chronicle.com/article/Behind-the-Webcams-Watchful/138505/

Kumar, R., Rosé, C. P., Wang, Y. C., Joshi, M., \& Robinson, A. (2007). Tutorial dialogue as adaptive collaborative learning support. Frontiers in artificial intelligence and applications.

Lehmann, E. L., \& Casella, G. (1998). Theory of point estimation (Vol. 31). Springer Science \& Business Media.

Li, X., Chang, K. M., Yuan, Y., \& Hauptmann, A. (2015, February). Massive open online proctor: Protecting the credibility of MOOCs certificates. InProceedings of the 18th ACM Conference on Computer Supported Cooperative Work \& Social Computing (pp. 1129-1137). ACM.

Linn, M. C., Gerard, L., Ryoo, K., McElhaney, K., Liu, O. L., \& Rafferty, A. N. (2014). Computer-guided inquiry to improve science learning. Science, 344(6180), 155-156.

LoSchiavo, F. M., \& Shatz, M. A. (2011). The impact of an honor code on cheating in online courses. MERLOT Journal of Online Learning and Teaching,7(2).

Mastin, D. F., Peszka, J., \& Lilly, D. R. (2009). Online academic integrity.Teaching of Psychology, 36(3), 174-178. 
McCabe, D. L., Butterfield, K. D., \& Trevino, L. K. (2012). Cheating in college: Why students do it and what educators can do about it. JHU Press.

McNutt, M. (2013). Bricks and MOOCs. Science, 342(6157), 402-402.

Palazzo, D. J., Lee, Y. J., Warnakulasooriya, R., \& Pritchard, D. E. (2010). Patterns, correlates, and reduction of homework copying. Physical Review Special Topics-Physics Education Research, 6(1), 010104.

Pappano, L. (2012). The Year of the MOOC. The New York Times, 2(12). Retrieved from http://www.nytimes.com/2012/11/04/education/edlife/massive-open-online-courses-are-multiplying-at-arapid-pace.html

Raines, D. A., Ricci, P., Brown, S. L., Eggenberger, T., Hindle, T., \& Schiff, M. (2011). Cheating in online courses: The student definition. The Journal of Effective Teaching, 11(2), 80-89.

Reich, J. (2015). Rebooting MOOC research. Science, 347(6217), 34-35.Smith, M. S. (2009). Opening education. Science, 323(5910), 89-93.

Stanford Online (2013). "Harnessing new technologies and methods to advance teaching and learning at Stanford and beyond." Stanford Online 2013 in Review.

Straumsheim, C. (2015, April 23).MOOCs for (a Year's) Credit. Inside Higher ED. Retrieved from https://www.insidehighered.com/news/2015/04/23/arizona-state-edx-team-offer-freshman-year-onlinethrough-moocs

Udacity. (2014, June 28). Udacity Terms of Service. Retrieved from https://www.udacity.com/legal/tos

Yang, D., Wen, M., Kumar, A., Xing, E. P., \& Rose, C. P. (2014). Towards an integration of text and graph clustering methods as a lens for studying social interaction in MOOCs. The International Review of Research in Open and Distributed Learning. 

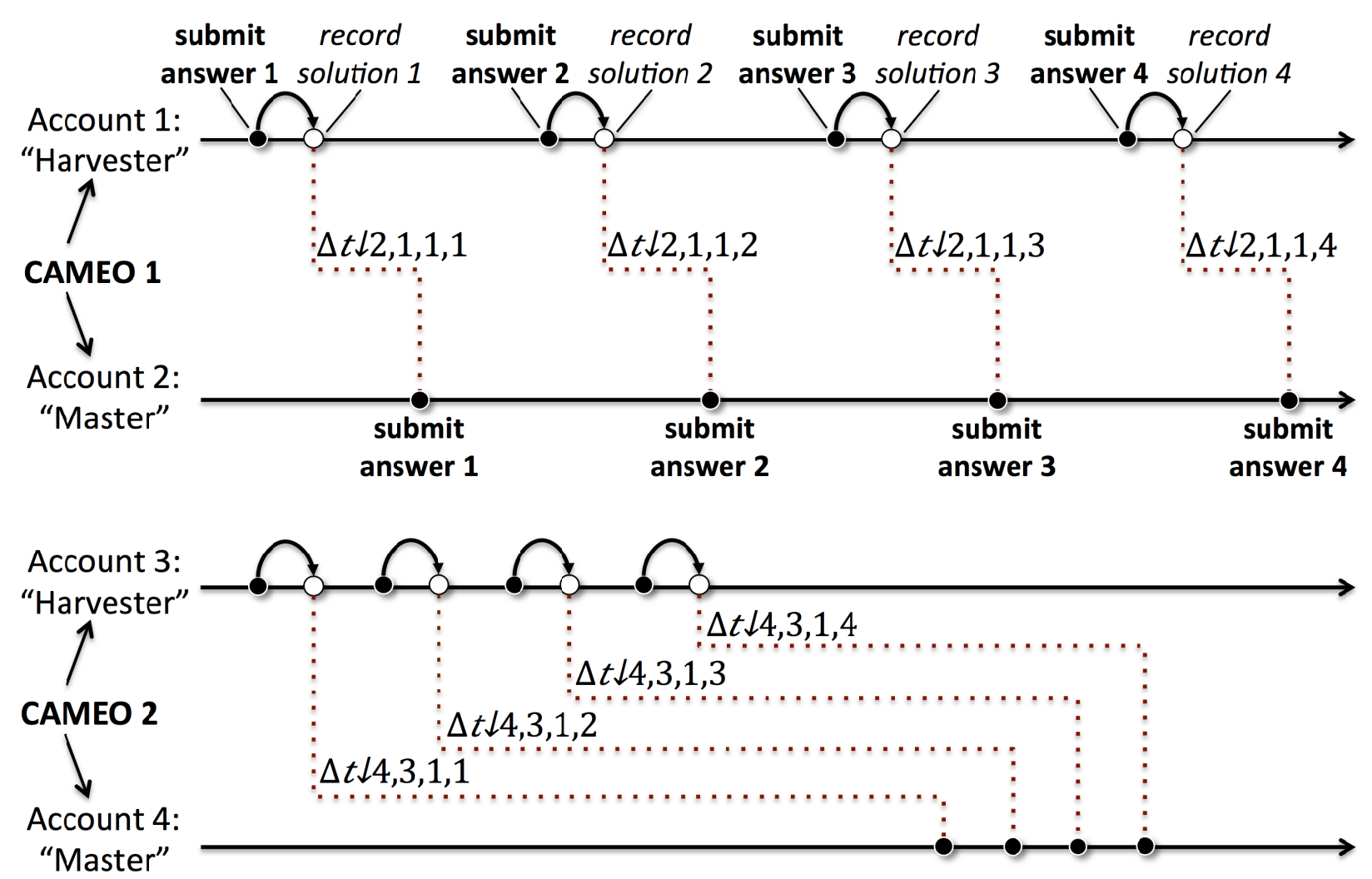

Fig 1. Two types of prototypical behavior when Copying Answers using Multiple Existences Online (CAMEO). A "harvester" account $h$ records correct solutions, and a "master" account $m$ submits correct answers. The time between harvesting in account $h$ (white dot) and correct answer submission by account $m$ (black dot) is estimable from the data and defined as $\Delta t_{m, h, c, i}$ for item $i$ in course $c$. The strategy employed by CAMEO 1 is to alternate harvesting and submission. The strategy of CAMEO 2 is to harvest a cluster and then submit a cluster. 


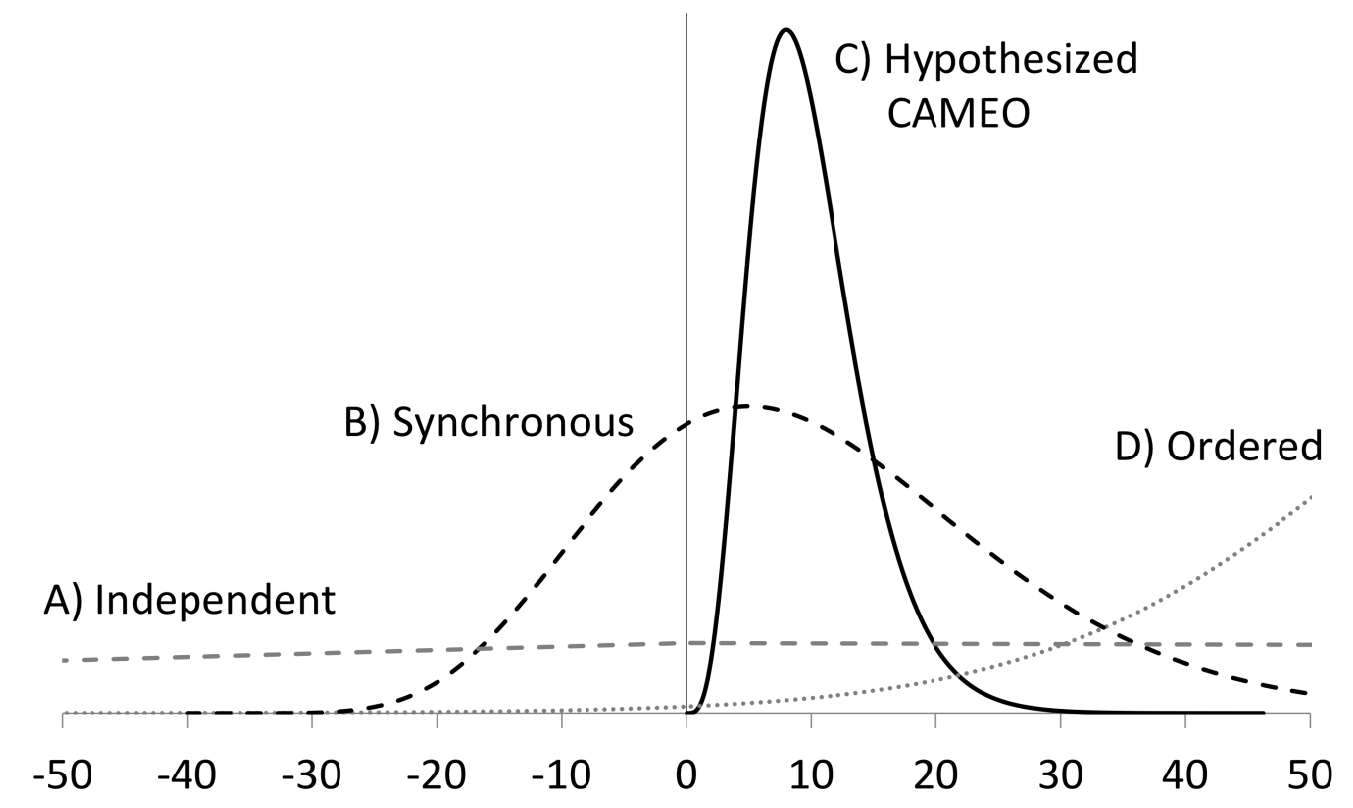

$\Delta t$ in Seconds (Candidate Master - Candidate Harvester)
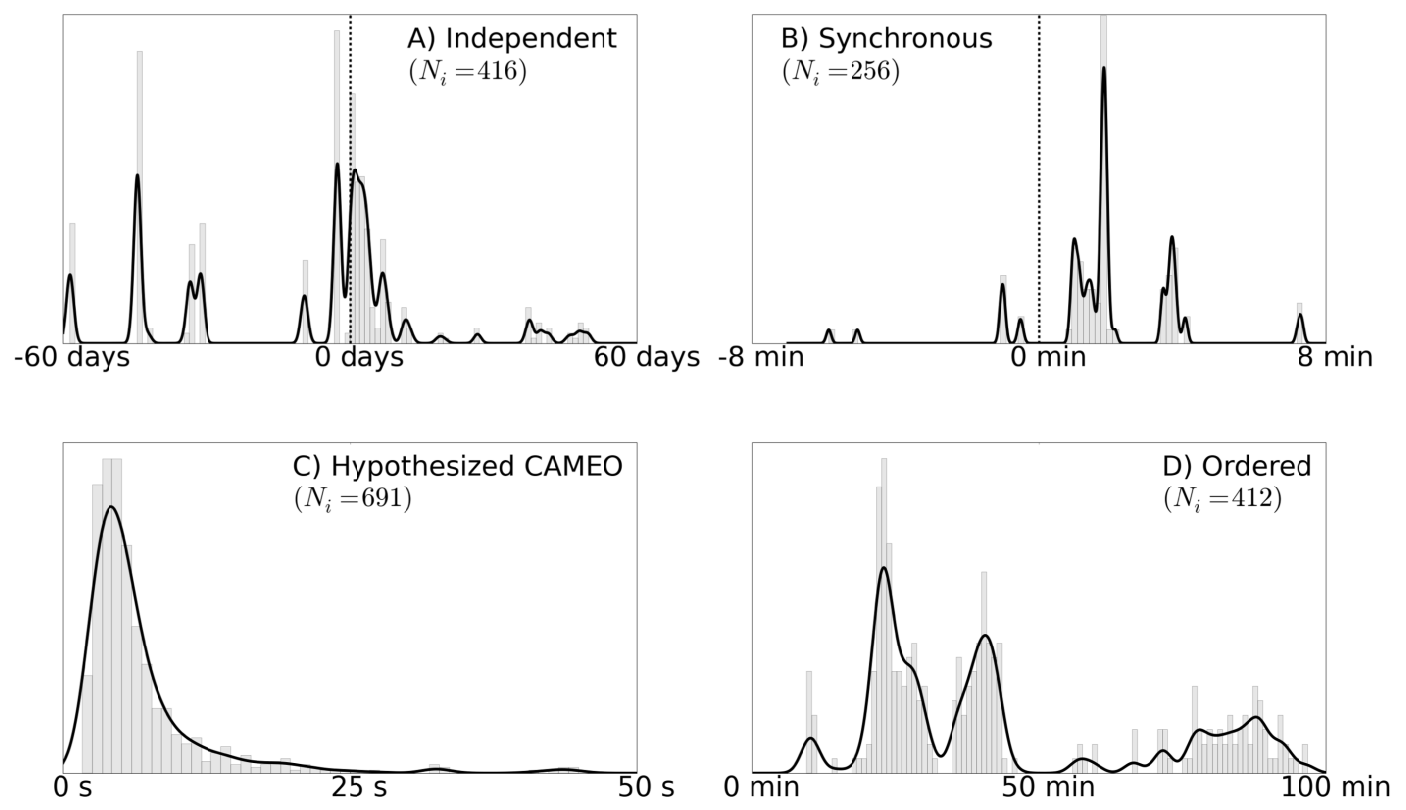

Fig 2. Four theoretical distributions of $\Delta t$ (top) illustrated by empirical distributions (below). Distribution A illustrates uniformly distributed "show answer" and "correct submission" times resulting in a shallow triangular distribution symmetrical around 0 . Distribution B illustrates synchronous submission with positive and negative $\Delta$ tvalues. Distribution $C$ illustrates prototypical "Copying Answers using Multiple Existences Online" (CAMEO) behavior, with candidate harvester accounts passing solutions to candidate master accounts over a short time span. Distribution D illustrates consistently and coincidentally ordered submissions over a longer 
time span. For the empirical distributions, the number of items shared between a harvester's "show answer" and a master's "correct submission" is displayed as $N_{i}$.

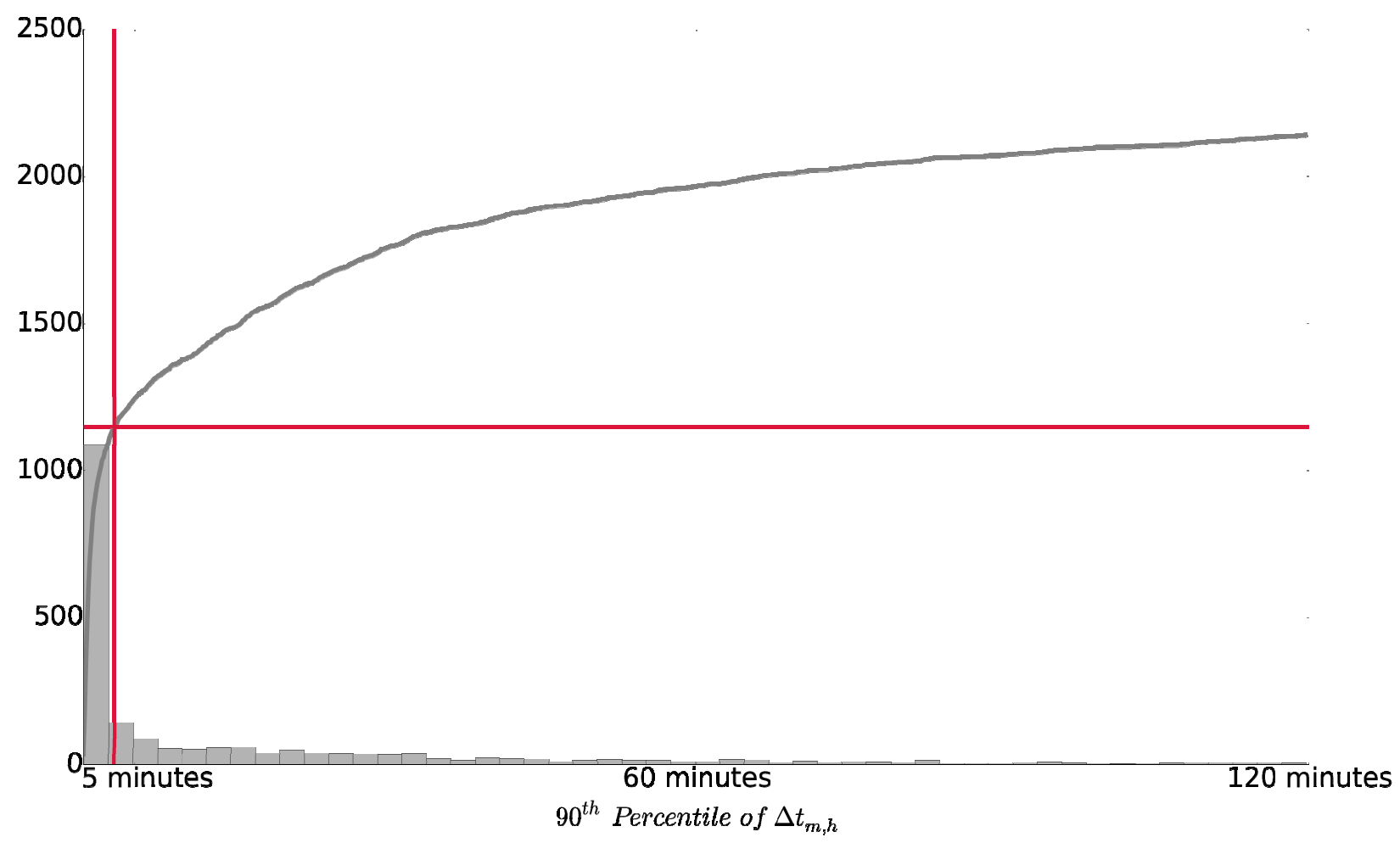

Fig. 3. Cumulative distribution (line) showing number of CAMEO users identified versus the $90^{\text {th }}$ percentile cutoff value of $\Delta t_{m, h}$ (Filter 2 in Table 1), together with the associated histogram (bars). The vertical red line depicts the cutoff value chosen; the horizontal red line is the corresponding number of CAMEO users identified. 
Table 1.A detection approach that asserts five necessary filtering conditions for candidate harvester $(\mathrm{CH})$ and candidate master $(\mathrm{CM})$ pairs to be classified as Copying Answers using Multiple Existences Online (CAMEO).

\section{Condition}

Explanation

The CH should harvest the correct
answer before the CM submits the
correct answer.
The CH should provide answers to the
$\mathrm{CM}$ quickly.

The $\mathrm{CH}$ should be guessing and uninterested in certification, whereas the goal of the CM is presumably certification.

4) The CM and $\mathrm{CH}$ should share an IP address or have shared one at some point in their course-taking history.

5) There should be few accounts that share or have shared an IP address with the $\mathrm{CM}$ and $\mathrm{CH}$.
This increases the likelihood that the $\mathrm{CM}$ and $\mathrm{CH}$ are in fact the same person.

This excludes internet cafes, school networks, and other common spaces where chance coincidence of $\Delta t$ may lead to false detection.
Operationalization

\author{
Bayesian $-90 \%$ confident that \\ the proportion of positive \\ $\Delta t$ values is $90 \%$. \\ The $90^{\text {th }}$ percentile of the \\ $\Delta t$ distribution should be less \\ than 5 minutes.
}

A CM must be certified. A CH must not be certified.

\author{
The $\mathrm{CM}$ and $\mathrm{CH}$ must share one \\ of the sets determined by the \\ transitive closure of modal IP \\ address and account name over \\ courses. \\ The number of accounts with a \\ shared modal IP address must \\ not exceed 10 .
}

Notes. The filters are chosen to be conservative, and their conjunctive application is more so, minimizing the chance of false identification at the cost of conceding missed CAMEO users. In terms of missed identification, Filter 1 excludes small-sample CAMEO users even when their proportions of positive times are $100 \%$. Filter 2 excludes CAMEO users that take more than 5 minutes to pass solutions between accounts. Filter 3 excludes those who use the CAMEO strategy but do not earn certificates. Filter 4 addresses those who use IP-masking strategies like the Tor browser. Filter 5 excludes CAMEO users within classrooms, cafes, and other scenarios in which IP addresses are shared. 


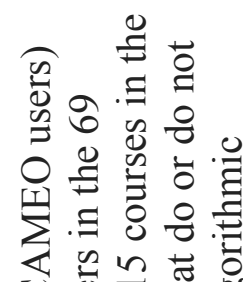

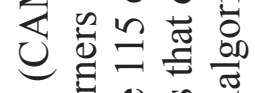

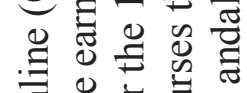

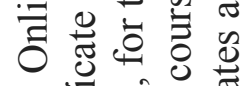

s.

彭 홍

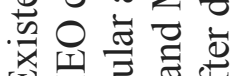

递药

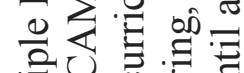

记

艺 。ี

이을

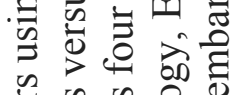

के की

空

过 0

.$\sum \sum 00$

중워

U

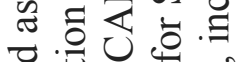

氖苛范

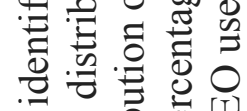

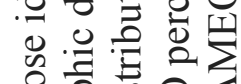

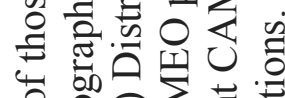

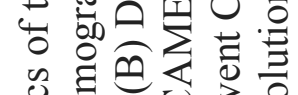

. ¿

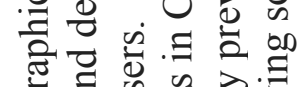

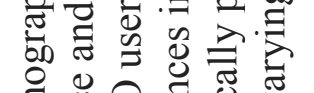

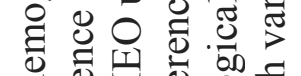

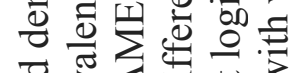

要还菏

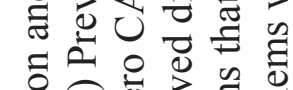

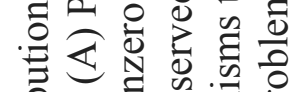

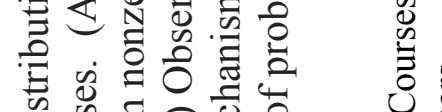

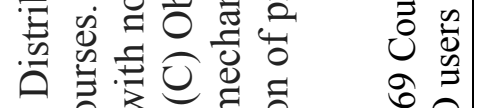

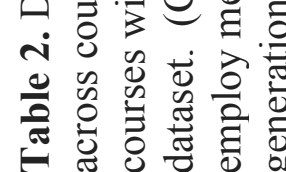

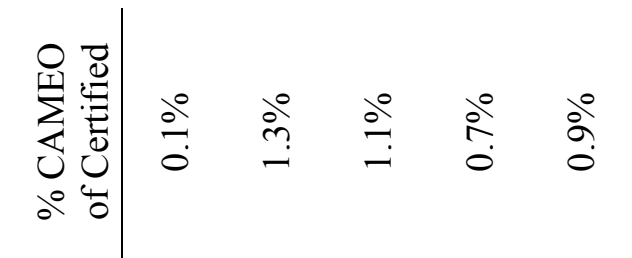

$\therefore \stackrel{\infty}{\sim} \underset{n}{ } \cong$

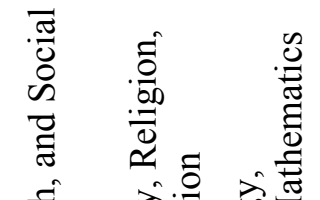

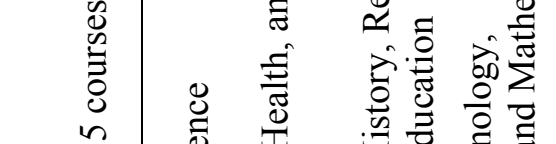

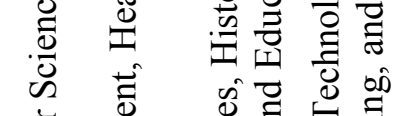

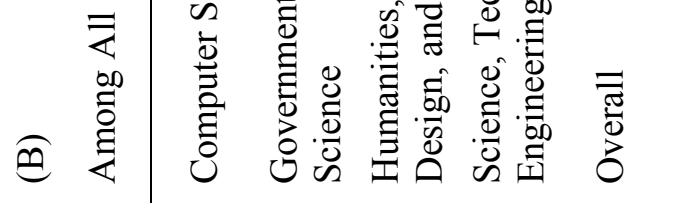

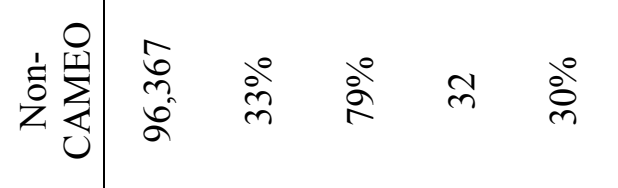

¿
กิ่

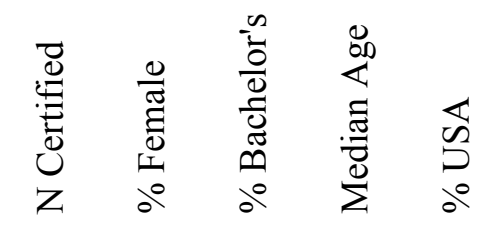

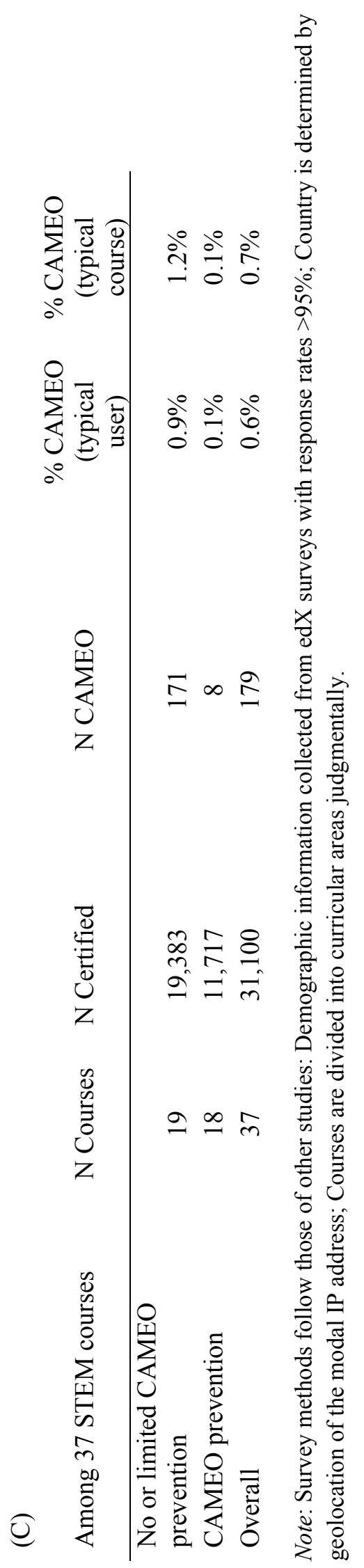


Table 3. Rates of Copying Answers using Multiple Certificates Online (CAMEO) among unique accounts earning multiple certificates.

\begin{tabular}{llll}
$\begin{array}{l}\text { Number of Certificates: } N \\
\text { (Lower Bound) }\end{array}$ & $\begin{array}{l}\text { Unique Certificate Earners with } \\
\geq N \text { Certificates: } M\end{array}$ & $\begin{array}{l}\text { Unique Certificate Earners, } M, \\
\text { with } \geq 1 \text { CAMEO }\end{array}$ & $\begin{array}{l}\text { Percent of Unique Certificate } \\
\text { Earners with } \geq 1 \text { CAMEO }\end{array}$ \\
\hline 1 & 103,370 & 657 & $1 \%$ \\
5 & 3,435 & 185 & $5 \%$ \\
10 & 1,262 & 82 & $6 \%$ \\
15 & 200 & 35 & $18 \%$ \\
20 & 73 & 18 & $25 \%$ \\
25 & 35 & 14 & $40 \%$ \\
30 & 15 & 7 & $47 \%$ \\
40 & 3 & 2 & $67 \%$
\end{tabular}

